\title{
Review
}

\section{Advances in the Treatment of Human Immunodeficiency Virus and Hepatitis B Virus Co-infection}

\author{
Guofang Sun
}

Department of Orthopaedics, Tianjin Medical University General Hospital, Tianjin China

\section{Keywords \\ HIV; HBV; Co-infection; ART \\ Correspondence \\ Guofang Sun, \\ E-mail: guofangs@163.com}

DOI: $10.1515 / i i-2017-0131$

\begin{abstract}
Hepatitis B virus (HBV) and human immunodeficiency virus (HIV) are transmitted through the same pathways. Therefore, the incidence of HBV in the HIV-infected population is higher than that in the healthy population, and is more obvious in China given the high HBV prevalence in the country. HIV and HBV co-infection can accelerate the disease process of HBV. Moreover, the incidence of cirrhosis and endstage liver disease is higher in patients co-infected with HIV and HBV than in patients infected HBV alone. When treating patients co-infected with HIV and HBV for HBV infection alone, care should be taken to avoid the induction of HIV resistance. HBV should be considered during drug selection for anti-retroviral treatment. Furthermore, the effective HBV treatment should be retained if anti-retroviral drugs require changing.
\end{abstract}

Infection with hepatitis B virus (HBV) is the main cause of chronic liver disease worldwide. HBV is transmitted through blood, sex, and maternal-infant channels. These transmission channels are consistent with that of the human immunodeficiency virus (HIV), and the efficiency of HBV transmission is higher than that of HIV ${ }^{[1]}$. Worldwide, $10 \%$ of HIV-infected individuals are co-infected with chronic $\mathrm{HBV}^{[2-3]}$. Given that China has a high incidence of hepatitis $B$, the incidence rate of domestic HIV patients co-infected with $\mathrm{HBV}$ is $14.6 \%{ }^{[4]}$. Chronic HBV associated with HIV develops into cirrhosis, end-stage liver disease, or hepatocellular carcinoma faster than pure $\mathrm{HBV}$ infection. In addition, because most HIV patients co-infected with $\mathrm{HBV}$ test positive for the hepatitis $\mathrm{B}$ virus e antigen ( $\mathrm{HBeAg}$ ) and some test positive for $\mathrm{HBeAg}$ seroconversion ${ }^{[5]}$, the risk of end-stage liver disease is higher among HIV patients coinfected with HBV than that among patients infected with HBV alone ${ }^{[6]}$. Therefore, the treatment and monitoring of HIV patients co-infected with chronic HBV should receive special attention. This paper provides a review of this aspect.

The incidence and mortality of AIDS, which is caused by HIV infection, have significantly decreased with the extensive application of anti-retroviral treatment (ART). ART has also significantly prolonged the average survival duration of HIV-infected individuals. However, ART is followed by increased hospitalization and fatality rates caused by non-AIDS diseases, such as liver disease caused by chronic HBV infection. Therefore, all HIV-infected individuals should undergo routine screening for the presence of $\mathrm{HBV}$ molecular markers, such as hepatitis $B$ virus surface antigen ( $\mathrm{HBs} A g$ ), hepatitis B virus core antibody ( $\mathrm{HBcAb})$, and hepatitis $B$ virus surface antibody (HBsAb). The ultimate goal for the treatment of patients co-infected with HIV and HBV infection is the same as that for the treatment of patients infected with HBV alone: to prevent progression and to reduce the incidence and mortality of HBV-related diseases. ART should be administered when the patients exhibit $\mathrm{HBV}$ infection 
activities, with manifest with increased transaminase, $\geq$ 2000IU/mL HBV deoxyribonucleic acid (DNA), or endstage liver disease with hepatic fibrosis ${ }^{[7]}$. Patients with severe liver disease and cirrhosis caused by hepatitis $B$ are required to take anti-HBV treatment.

Currently available drugs for $\mathrm{HBV}$ infection include interferon, pegylated interferon, lamivudine (3TC), adefovir dipivoxil (ADV), entecavir (ETV), and telbivudine (LDT). Tenofovir disoproxil fumarate (TDF) and emdtricitabine (ETC) have been approved for HIV treatment and exert therapeutic effects on HBV infection. The best treatment endpoint for $\mathrm{HBV}$ infection is negative HBsAg and the occurrence of HBsAb. Few HIV-infected patients, however, reach these treatment end points. More realistic treatment goals include the long-term suppression of $\mathrm{HBV}$, the reduction of liver inflammation, and the suspension or delay of liver fibrosis. For HIV patients with HBV infection, the first problem is the indication of ART. $\mathrm{CD}_{4+} \mathrm{T}$ lymphocyte $\left(\mathrm{CD}_{4}\right.$ cell) levels are $<350 / \mu \mathrm{L}$ in individuals infected with HIV alone and $<500 / \mu \mathrm{L}$ in HIV patients with HBV co-infection. When $\mathrm{CD}_{4}$ cell levels are $<500 / \mu \mathrm{L}, \mathrm{ART}$ should be considered as a treatment option. The current guidelines for the treatment of HIV infection in China recommend that ART should be initiated when hepatitis occurs regardless of $\mathrm{CD}_{4}$ cell levels ${ }^{[8]}$. The specific treatment process has the following two situations.

\section{HIV and HBV Co-Infected Patients with the Long-term Non-Progression of HIV Infection and Reluctant to Initiate ART}

1) In this case, the specific medication ${ }^{\left[{ }^{[-10]}\right.}$ is pegylated interferon. The desired therapeutic effects in patients are high level of alanine transaminase (ALT), low level of $\mathrm{HBV}-\mathrm{DNA}$, and positive $\mathrm{HBeAg}$. For the treatment of cirrhosis patients co-infected with HIV and HBV, pegylated interferon should be avoided and telbivudine should be used alone instead. If HBV-DNA exceeds the detection limit at 24 weeks of treatment, ADV can be added to decrease the risk of drug resistance. Drug users with the early initiation of ART should be treated with a drug combination that includes TDF and LAM/ FTC.
2) Plasma HBV-DNA level is significantly related with the risk of hepatitis B cirrhosis and hepatocellular carcinoma. Therefore, the possibility of hepatitis activity remains in patients with high HBV-DNA level and normal ALT level. Additional measures should be taken to assess the extent of liver inflammation. These measures include liver biopsy or non-invasive examination, such as plasma liver fibrosis markers or transient liver elastic scanning (Fibroscan). Liver biopsy scoring systems can be used as a reference for the suitability of a specific treatment. For example, scores >A2 and/or F2 in the METAVIR system indicate that anti-HBV therapy should be provided via the above-mentioned therapeutic schemes.

3) When used to treat HIV patients with HBV infection without the initiation of ART, ETV can induce M184V mutations and then generate lamivudine and emdtricitabine resistance. Thus, ETV cannot be used alone for the treatment of HBV patients with HIV infection ${ }^{[11]}$.

\section{HIV/HBV Co-Infected Patients with the Initiation of ART or in the Process of ART}

1) Although $\mathrm{HBV}$ is not resistant to lamivudine, patients who have ever taken lamivudine should be treated with ART that includes TDF and LAM/FTC ${ }^{[12-13]}$ to delay $\mathrm{HBV}$ resistance to LAM or FTC. For patients with HIV and $\mathrm{HBV}$ co-infection, the generation of lamivudine resistance in $\mathrm{HBV}$ is faster than that in pure $\mathrm{HBV}$ infection, and is approximately $50 \%$ in 2 years and $90 \%$ in 4 years ${ }^{[14]}$. If $\mathrm{HBV}$ patients with HIV infection have received ART of LAM combined with TDF for 48 weeks, HBV load continues to exceed the lower limit of detection, and LAM/FTC resistance or HBV rebound is absent, then the patients can achieve effective HBV suppression under the original regimen without immediate treatment with ETV ${ }^{[15]}$.

2) If there is $\mathrm{HBV}$ resistance to lamivudine, TDF should be added or used instead of lamivudine in ART. HBV-DNA cannot be completely suppressed by the combination of TDF and LAM, although theoretically, the combination of TDF and ETV can be used to enhance the efficacy of anti-HBV treatment. However, this effect 
remains unconfirmed by clinical studies. Patients with Fanconi syndrome after TDF should receive tenofovir disoproxil fumarate combined with probenecid to decrease the renal toxicity associated with TDF ${ }^{[16]}$. Alternatively, ETV can be added to ART, and the dosage should be adjusted from $0.5 \mathrm{mg}$ per day to $1 \mathrm{mg}$ per day. ETV has cross-resistance to TDF and FTC; thus, HBVinfected individuals may become resistant to ETV. Therefore, when these patients are treated with ETV, the level of HBV-DNA should be closely monitored (once every 3 months) to determine HBV breakthrough as soon as possible. TDF should be added or used instead of ETV in treating ETV-resistant patients. ETV can be added to the treatment of TDF-resistant patients. However, HBV is not resistant to TDF in vitro.

3) If ART is only effective against HBV because of HIV resistance, the drug with efficacy against HBV should be retained even when other drugs in ART are changed.

Medication course: Patients should receive pegylated interferon once a week for 48 weeks; nucleoside reverse transcriptase inhibitor (LDT and ADV) cannot be suspended until $\mathrm{HBsAg}$ is negative for 6 months. LDT and ADV should be suspended under close monitoring if ART is not initiated under the following conditions: if the patient turns from $\mathrm{HBeAg}(+)$ to $\mathrm{HBeAb}(+)$ for more than 6 months or HBsAg disappears; and $\mathrm{HBsAb}(+)$ lasts for more than 6 months or $\mathrm{HBs} \mathrm{Ab}(+)$ appears after the treatment of original $\mathrm{HBeAg}(-)$ for more than 6 months. If the patient has initiated ART and the nucleoside reverse transcriptase inhibitors have to be replaced, anti-HBV drugs must be stopped under close monitoring to avoid the rebound effect. In addition, patients with combined infections should be treated with long-term anti-HBV therapy. The relapse rate is approximately $30 \%{ }^{[17-18]}$ when the drugs are prematurely stopped before the appropriate time. Relapse after drug withdrawal may exacerbate the damage to liver function and even result in hepatic decompensation. In case of relapse, the original anti $\mathrm{HBV}$ treatment should be added immediately.

Therapeutic effect evaluation ${ }^{[19]}$ : 1) Primary nonresponse. After 12 weeks of treatment, the amplitude of HBV-DNA load should decrease by <1log10. 2) Complete response. After 24 weeks of treatment, HBV-DNA load should be lower than the detection limit in the RT-PCR test (HBV-DNA being lower than the lower limit at 24 weeks indicates the low incidence of drug resistance ${ }^{[20]}$ ); 3) Partial response: HBV-DNA decreases to $>1 \log 10$ at 24 weeks but remains higher than the detection limit. 4) Sustained response. During treatment, the viral load is lower than the detection limit. Six months after drug withdrawal, the viral load remains below the lower limit of detection. After 12 weeks of treatment, for patients good compliance but no primary response or whose HBV-DNA load increases by $>1 \log 10$ compared with the lowest level of viral load at 12 weeks of treatment, genetic resistance tests should be carried out after the confirmation of good compliance. When LAM resistance exists, FTC and LDT should be avoided and TDF can be added ${ }^{[12,21]}$. The dosage of nucleoside antiviral drugs should be carefully adjusted for patients with renal impairment.

Follow-up review: After the initiation of anti-HBV therapy, HBV-DNA levels should be reviewed every 12 weeks to assess the efficacy of antiviral treatment. For patients who are positive for E antigen, HBeAg levels should be reviewed every 6 months. The regular detection of $\mathrm{HBV}-\mathrm{DNA}$ and the quantification of $\mathrm{HBeAG}$ and $\mathrm{HBeAb}$ should improve the prognosis of patients with possible HBeAG seroconversion. ALT level should be closely monitored and reviewed once a month during antiHBV treatment. If ALT level stabilizes within 6 months, the review can be extended to once every 3 months. Given that chronic hepatitis B itself is an immune-mediated disease, immune reconstitution may be accompanied by increased transaminase levels after the initiation of antiHIV and/or anti-HBV. The application of TDF+FTC ART can decrease the risk for immune reconstitution associated with chronic hepatitis $\mathrm{B}^{[22]}$. After the initiation of ART, immunologic functions are reconstructed, possibly leading to $\mathrm{HBV}$-related hepatitis activation, which manifests as increased $\mathrm{CD}+4 \mathrm{~T}$ lymphocytes and ALT after treatment. Therefore, liver function should be closely monitored after ART. Significantly increased ALT levels indicate jaundice and damage to liver synthetic function. Thus, a consultation with a liver specialist is needed. In addition, some ART medication may also elevate liver transaminase. The elevation of liver transaminase caused by this factor is more common in HBV associated with HIV infection ${ }^{[23]}$. This kind of 
damage is negligible and drug suspension and special treatment are not needed. However, when ALT increases to 5-10 times the upper limit of normal (ULN), drugs for liver treatment should be actively sought. If the patients exhibit allergic reactions (fever and rash) and hepatitis symptoms (nausea, vomiting, abdominal pain, and jaundice), the suspected medications should be stopped. Elevated liver enzymes in HIV patients with $\mathrm{HBV}$ infection indicate $\mathrm{HBeAg}$ seroconversion. Increased ALT during ART may indicate a combination of remaining viral infection, such as hepatitis $A$, hepatitis $C$, hepatitis $\mathrm{E}$, and alcoholic and non-alcoholic fatty liver diseases. Therefore, the cause of the increase in liver enzymes must be first identified before considering the suspension of the suspected drugs.

\section{Declarations}

\section{Acknowledgements}

No.

\section{Competing interests}

The author declares that she has no competing interest.

\section{Authors' contributions}

GF Sun made the literature analysis and wrote, discussed and revised the manuscript of this review.

\section{References}

1 Lee WM. Hepatitis B virus infection. N Engl J Med,1997,337(24):173 3-1745.

2 Thio CL. Hepatitis $B$ and human immunodeficiency virus coinfection. Hepatology, 2009, 49 (5Suppl): S138-145.

3 Kourtis AP, Bulterys M, Hu DJ, et al. HIV-HBV coinfection aglobal challenge. N Engl J Med, 2012, 366(19): 1749-1752.

4 Wang $\mathrm{H}$, Li Y, Zhang C, et al. Immunological and virological responses to cART in $\mathrm{HIV} / \mathrm{HBV}$ co-infected patients from a multicenter cohort. AIDS, 2012, 26(14): 1755-1763.

5 Colin JF, Cazals-Hatem D, Loriot MA, et al. Influence of human immunodeficiency virus infection on chronic hepatitis $B$ in homosexual men. Hepatology, 1999, 29(4):1306-1310.

6 Thio CL, Seaberg EC, Skolasky RJ, et al. HIV-1, hepatitis B virus, and risk of liver-related mortality in the Multicenter Cohort Study (MACS). Lancet, 2002, 360(9349):1921-1926.
7 Lok AS, McMahon BJ. Chronic hepatitis B: update 2009. Hepatology, 2009, 50(3): 661-662.

8 AIDS clinical expert group of Health and Family Planning Commission. "Standard of antiviral therapy for adult / adolescent AIDS patients"

9 Liaw YF, Kao JH, Piratvisuth T, et al. Asian-Pacific consensus statement on the management of chronic hepatitis B: a 2012 update. Hepatol Int, 2012, 6(3): 531-561.

10 Keeffe EB, Dieterich DT, Han SH, et al. A treatment algorithm for the management of chronic hepatitis B virus infection in the United States: 2008 update. Clin Gastroenterol Hepatol, 2008, 6(12): 1315-1341.

11 McMahon MA, Jilek BL, Brennan TP, et al. The HBV drug entecavireffects on HIV-1replication and resistance. N Engl J Med, 2007, 356(25): 2614-2621.

12 Matthews GV, Seaberg E, Dore GJ, et al. Combination HBV therapy is linked to greater HBV DNA suppression in a cohort of lamivudineexperienced HIV/HBV coinfected individuals. AIDS, 2009, 23(13): 1707-1715.

13 de Vries-Sluijs TE, Reijnders JG, Hansen BE, et al. Long-term therapy with tenofovir is effective for patients co-infected with HIV and HBV. Gastroenterology, 2010, 139(6): 1934-1941.

14 Benhamou $\mathrm{Y}$, Bochet $\mathrm{M}$, Thibault V, et al. Long-term incidence of hepatitis B virus resistance to lamivudine in human immunodeficiency virus-infected patients. Hepatology, 1999, 30(5): 1302-1306.

15 Childs K, Joshi D, Byrne R, et al. Tenofovir-based combination therapy for HIV/HBV co-infection. AIDS, 2013, 27(9): 1443-1448.

16 Izzedinea $\mathrm{H}$, Thibault V, Valantinc MA, et al. Tenofovir/probenecid combination in $\mathrm{HIV} / \mathrm{HBV}$-coinfected patients: how to escape Fanconi syndrome recurrence. AIDS, 2010, 24(7): 1077-1090.

17 Dore GJ, Soriano V, Rockstroh J, et al. Frequent hepatitis B virus rebound among HIV-hepatitis B virus-coinfected patients following antiretroviral therapy interruption. AIDS, 2010, 24(6): 857-865.

18 Bellini C, Keiser O, Chave JP, et al. Liver enzyme elevation after lamivudine withdrawal in HIV-hepatitis B virus coinfected patients: the Swiss HIV Cohort Study. HIV Med, 2009, 10(1): 12-18.

19 European Association For The Study Of The Liver. EASL Clinical Practice Guidelines: Management of chronic hepatitis B virus infection. J Hepatol, 2012, 57(1):167-185.

20 Zeuzem S, Gane E, Liaw YF, et al. Baseline characteristics and early on-treatment response predict the outcomes of 2years of telbivudine treatment of chronic hepatitis B. J Hepatol, 2009, 51(1): 11-20.

21 Vassiliadis TG, Giouleme O, Koumerkeridis G, et al. Adefovir plus lamivudine are more effective than adefovir alone in lamivudineresistant $\mathrm{HBeAg-chronic} \mathrm{hepatitis} \mathrm{B} \mathrm{patients:} \mathrm{a} \mathrm{4-year} \mathrm{study.} \mathrm{J}$ 
Gastroenterol Hepatol, 2010, 25(1): 54-60.

22 Panel on antiretroviral guidelines for adults and adolescents. Guidelines for the use of antiretroviral agents in HIV-1-Infected adults and adolescents [EB/OL] http://aidsinfo.nih.gov/guidelines, 2014-05-02.
23 Sulkowski MS, Thomas DL, Chaisson RE, et al. Hepatotoxicity associated with antiretroviral therapy in adults infected with human immunodeficiency virus and the role of hepatitis C or B virus infection. JAMA, 2000, 283(1): 74-80. 